\title{
Identificando requerimientos para el diseño de un aplicativo móvil de Realidad Aumentada para el aprendizaje del patrimonio histórico regional
}

\author{
Lizeth M. Zambrano ${ }^{1}$, Greis Francy M. Silva-Calpa ${ }^{2}$, José L. Romo ${ }^{1}$ \\ ${ }^{1}$ Licenciatura en Informática, Universidad de Nariño, Pasto (N) - Colombia \\ ${ }^{2}$ Instituto Tecgraf/Pontifícia Universidade Católica do Rio de Janeiro - Brasil \\ sedecrem1991@udenar.edu.co, greis@tecgraf.puc-rio.br, rhomojose@udenar.edu.co
}

\begin{abstract}
Augmented reality (AR) applications intended for historical and cultural heritage knowledge have been focused mostly on the promotion of tourism; for this reason, it is important to carry out research focused on the learning of regional heritage through contextualized applications to the region and the academic field. This study aims to identify a set of requirements for the design of AR mobile applications for learning regional historical heritage. Based on a literature review, surveys with social science teachers, the development of an AR mobile application, and its heuristic evaluation, a set of requirements is proposed as a contribution to future research.
\end{abstract}

Resumen. Aplicaciones en realidad aumentada $(R A)$ para la aproximación al patrimonio histórico y cultural, se han centrado, en su mayoría, en el fomento al turismo; por ello, se hace importante adelantar investigaciones orientadas al aprendizaje del patrimonio regional mediante aplicaciones contextualizadas a la región y al campo académico. Este estudio pretende identificar un conjunto de requerimientos para el diseño de un aplicativo móvil de RA para el aprendizaje del patrimonio histórico regional. A partir de una revisión bibliográfica, encuestas con docentes del área, el desarrollo de un aplicativo móvil en $R A$ y su respectiva evaluación heurística, se propone un conjunto de requerimientos como contribución para futuras investigaciones.

\section{Introducción}

El aprendizaje del patrimonio histórico le otorga a la población la posibilidad de valorar y afirmar su identidad cultural, dado que la historia de una región es la herencia de sus antepasados en las nuevas generaciones; sin embargo, cuando se desea hablar de la historia regional y local, esta suele ser desconocida [Moran and Narvaez 2015]. En las regiones pequeñas, cuando de historia se trata, no sólo los textos la cuentan, los monumentos situados a lo largo del territorio también lo hacen, pero pasan inadvertidos la mayor parte del tiempo, al estar ubicados con poca o ninguna información histórica de utilidad. Como lo afirma [Moran and Narvaez 2015] “... un ciudadano promedio tiene más accesibilidad a fotografías e historias de lugares muy lejanos geográficamente, y no puede acceder tan fácilmente a la información de la iglesia que visita todos los domingos", provocando que se tenga en mayor medida la influencia extranjera que de alguna forma reemplaza la legitima cultura de una región auténtica [Mejía et al. 2012] y afectando, por tanto, el adecuado aprendizaje del patrimonio local.

Para aportar a la solución de dicha problemática, se consideran las nuevas tecnologías, que hoy en día se han convertido en aliadas de la comunidad educativa. Un ejemplo de estas es la Realidad Aumentada (RA) como apoyo en el aprendizaje, que ofrece la posibilidad de enriquecer un entorno real, gracias a diversos objetos virtuales sobrepuestos a él, sin requerir, 
X Congresso Brasileiro de Informática na Educação (CBIE 2021)

Anais do XXXII Simpósio Brasileiro de Informática na Educação (SBIE 2021)

necesariamente, que el usuario [estudiante] se ubique físicamente en un lugar en específico [Zuñiga 2008]. Si bien es cierto que existen algunos estudios sobre el desarrollo de aplicativos móviles de RA a nivel regional, en su mayoría los productos son pensados como software destinado al fomento del turismo, dejando de lado la historia regional y los fines educativos. Ahora bien, para el desarrollo de aplicativos en RA orientados a contribuir al aprendizaje del patrimonio histórico regional, los fundamentos educativos toman importancia, además de la forma cómo la tecnología es usada y adecuada para tal fin. Por tanto, para que una aplicación móvil se use con fines académicos, debe estar orientada al sector educativo desde el momento del diseño [Luna et al. 2019], pues un diseño apropiado puede contribuir a que los estudiantes adquieran conocimientos de forma más eficiente [Fraga and de Menezes 2017]. En ese sentido, el objetivo de este estudio consiste en identificar un conjunto de requerimientos para el diseño de una aplicación móvil de RA que contribuya en el aprendizaje del patrimonio histórico regional.

Para alcanzar el objetivo planteado, tomamos como caso de estudio la ciudad de Pasto, ubicada al sur de Colombia, conocida mundialmente por su carnaval de negros y blancos ${ }^{1}$; fue escogida porque sus eventos culturales son reconocidos nacionalmente, pero muchas veces se desconocen nombres y biografías de personalidades de la ciudad, así como causas y efectos del actuar histórico de esta región, al no considerarse relevante para el resto del país, sumado a que la información histórica de la ciudad no se encuentra en lugares de acceso regionales, sino en bibliotecas de ciudades principales del país o hasta en países vecinos [Mejía et al. 2012].

Considerando la problemática sobre el aprendizaje del patrimonio histórico regional, este estudio sigue el enfoque Design Science Research (DSR) como método de investigación, puesto que objetiva el desarrollo de un dispositivo, que en este caso es el aplicativo móvil en RA, para un determinado contexto (la ciudad seleccionada), así como contribuye al saber científico desde la propuesta de un conjunto de requerimientos para apoyar el diseño de este tipo de productos [Pimentel et al. 2019]. Para ello se realizaron las siguientes etapas de investigación:

Inicialmente se hizo una revisión bibliográfica de trabajos relacionados con el diseño de aplicaciones de RA orientadas al patrimonio regional, donde se incluyeron investigaciones de orden internacional, nacional y local que permitieron identificar algunos requerimientos particulares abordados por esos estudios. En la misma línea, se realizó la revisión documental correspondiente y una etapa de elicitación de requisitos, que dieron lugar a soportar en la práctica los requerimientos encontrados en la teoría. Con los requerimientos identificados, se procedió al diseño del aplicativo móvil de RA e implementación de un módulo, que fue evaluado por inspección, utilizando el conjunto de heurísticas de Nielsen [Nielsen 1995] con el fin de identificar problemas de usabilidad desde el entender educativo. Finalmente, se presenta un consolidado de los requerimientos identificados en el desarrollo de las etapas anteriores.

Si bien, lo presentado muestra características específicas del caso de estudio, la contribución principal es la propuesta del conjunto de requerimientos que pueden ser útiles para otros investigadores en el diseño de aplicativos en RA para fomentar el patrimonio regional; además, se pretende incentivar nuevos y más estudios sobre el aporte de tecnologías, dirigidos a espacios regionales y ciudades no principales, que cuentan con un excelente patrimonio histórico.

\section{Trabajos Relacionados}

Desde los estudios en la literatura acerca de aplicaciones de RA para el fomento del patrimonio [Torres 2011, Bonifaz and Molina 2015, Bekele et al. 2018, Aliprantis and Caridakis 2019, Cardenas 2020], este trabajo se enfoca en aquellos relacionados con el aprendizaje del patrimo-

\footnotetext{
${ }^{1}$ https://carnavaldepasto.org/
} 
X Congresso Brasileiro de Informática na Educação (CBIE 2021)

Anais do XXXII Simpósio Brasileiro de Informática na Educação (SBIE 2021)

nio histórico regional, destacando los requerimientos mencionados por algunos de los autores para el desarrollo de tales aplicaciones.

[Suárez 2017] propone el aplicativo GeoHistoryApp que, usa RA basada en geolocalización para complementar y facilitar el aprendizaje de conceptos históricos regionales impartidos en el aula de clase. El autor evidencia que el desconocimiento de los hechos históricos a nivel regional llevan a "...falta de identidad, de sentido patrio y de pertenencia... ” [Suárez 2017]. Destaca un conjunto de requerimientos funcionales y no funcionales, tales como la implementación de marcadores, etiquetas y uso de la cámara del dispositivo, los cuales le permiten al usuario visualizar información en tiempo real en el lugar donde ocurrido el hecho histórico, acompañado de una descripción y fotografías antiguas. También, se destaca el uso de marcadores [Martinez Carrillo et al. 2021] , puesto que estos le otorgan al estudiante la oportunidad de interactuar con los objetos desde distintas perspectivas y posiciones. Sin embargo, se resalta que, si bien la RA le da un valor agregado al aprendizaje del patrimonio histórico, uno de los principales problemas para su uso es la falta de capacitación de los docentes.

Por su parte, [Luna et al. 2019] realizaron una revisión de ocho (8) aplicaciones de RA para la enseñanza y aprendizaje del patrimonio en regiones de España. El estudio muestra que la mayoría de los aplicativos revisados no fueron evaluados por expertos en el campo educativo, además, para su funcionamiento requieren que el usuario se sitúe en el espacio patrimonial; también se encontró que estas aplicaciones no se adaptan a diferentes edades, niveles y capacidades cognitivas, por lo cual su ciclo de vida es corto. Para subsanar estas dificultades, los autores destacan la importancia de desarrollar aplicaciones que tengan una adaptación educativa apropiada, además de permitir usarlas en diferentes lugares, sin estar físicamente en el espacio patrimonial [Luna et al. 2019]. Aunque el estudio en mención no se centra en la identificación de requerimientos, deja entrever la necesidad del desarrollo de aplicativos móviles orientados al aprendizaje histórico, que a su vez sean más versátiles y flexibles.

[Hincapié et al. 2020] desarrollaron una aplicación para la reactivación y la enseñanza del patrimonio histórico de la plaza Cisneros en Medellín. Para el desarrollo, los autores usaron fotografías históricas y realizaron entrevistas con quienes habitaron el lugar durante esos años. Los autores destacan que la RA permite recrear elementos desaparecidos del patrimonio cultural en el sitio geográfico donde alguna vez estaban ubicados. Si bien el estudio de [Nagata et al. 2017] es orientado hacia la percepción del aprendizaje, la selección de requisitos de los autores se basó principalmente en lo existente actualmente en materia de RA y mapas de navegación. Los autores recomiendan verificar el software en tres puntos: la conectividad, verificación de los niveles de precisión de los dispositivos usados (tablets) y la corrección de errores que surgen de la visualización de datos.

En el estudio de [Rodríguez et al. 2016] se indagó acerca del uso de tecnologías emergentes para el aprendizaje y apropiación patrimonial de la ciudad de Cartagena; una de las conclusiones de los autores fue destacar el uso de la RA en diferentes campos, entre ellos el educativo. En su estudio, los autores evidenciaron inconvenientes debido a la arquitectura del lugar y problemas de conexión a Internet, estos se constituyen en elementos clave a tener en cuenta al momento de utilizar aplicaciones basadas en RA para visualizar obras y monumentos. En este sentido, uno de los requerimientos que no debe obviarse, es la disponibilidad de información offline, para que un usuario pueda disponer de gran parte del contenido histórico y recursos multimedia del aplicativo móvil, independientemente de su condición de conectividad.

Apenas dos estudios [Meneses and Montenegro 2015] y [Moran and Narvaez 2015] proponen sistemas en RA para promover los atractivos turísticos de la ciudad de Pasto 
X Congresso Brasileiro de Informática na Educação (CBIE 2021)

Anais do XXXII Simpósio Brasileiro de Informática na Educação (SBIE 2021)

[Meneses and Montenegro 2015] destacan como requerimientos, la selección cuidadosa de los sitios turísticos de la ciudad, la medición de su popularidad desde los ámbitos religioso, cultural y político, la relevancia del diseño de los escenarios en 3D, así como el desarrollo de los recursos multimedia utilizando herramientas pertinentes.

\section{Metodología}

El estudio, al centrarse en el diseño y desarrollo de un aplicativo móvil en RA para contribuir a la solución de una problemática contextual y aportar al conocimiento científico, se fundamentó en lo cualitativo bajo el enfoque Design Science Research (DSR) [Pimentel et al. 2019]. La metodología adoptada incluye las etapas consolidadas por [Dresch et al. 2015]:

- Revisión de la literatura y sugerencias de posibles soluciones. Se identificó los requerimientos indicados por los autores desde la revisión de la literatura (Sección 2). Luego, se realizó una Elicitación de requisitos, para proponer nuevos requerimientos, desde la encuesta con docentes de instituciones educativas de Pasto (Sección 4.1).

- Implementación. A partir de los requerimientos identificados, se prosigue con el diseño e implementación de un aplicativo móvil, siguiendo la metodologia propuesta por [Zuñiga 2008] que cubre las necesidades específicas para el desarrollo de aplicaciones en RA, como esta detallado en la Sección 4.2 y Sección 4.3.

- Evaluación. El aplicativo móvil es evaluado por inspección con las reglas Heurísticas de Nielsen [Nielsen 1995]. La labor la hizo un profesional en educación e informática, con miras a identificar problemáticas de usabilidad desde el punto de vista del aprendizaje e interacción con el sistema. La evaluación y resultados se presentan en la Sección 4.4

- Reflexión y aprendizaje. Los resultados, analizados y discutidos, llevan a la consolidación de un conjunto de requerimientos como aporte de investigación, presentados en la Sección 5.

\section{Aplicativo móvil de RA para el aprendizaje del patrimonio regional}

Para culminar el desarrollo del aplicativo móvil, se tienen en cuenta 3 apartados: la Subsección 4.1 Elicitación de requisitos, la Subsección 4.2 El diseño del aplicativo móvil y la la Subsección 4.3 Implementación del mismo, que se detallan con mayor profundidad a continuación.

\subsection{Elicitación de Requisitos}

Etapa fundamental orientada a subsanar los requerimientos no funcionales que otorgarán el contexto necesario al aplicativo móvil. Para tal fin, se constituyen en fuente primaria la información recolectada de libros, los relatos históricos y las opiniones de los docentes del área de Ciencias Sociales de diferentes instituciones educativas de Pasto. Participaron siete (7) docentes quienes, después de dar su consentimiento informado, respondieron una encuesta semiestructurada, elaborada en Google Forms, que recopiló información general (edad, institución educativa) y específica que permite seleccionar los lugares, monumentos, hechos y personajes históricos de la región, los cuales se considera deberían estar disponibles en el aplicativo móvil; se obtuvo también información que posibilita identificar los grados escolares y los Derechos Básicos de Aprendizaje (DBA) que abordan la historia regional, lo cual dá el énfasis educativo al aplicativo.

Con esta información, se pudo evidenciar que la población encuestada está conformada por docentes entre los 30 y 60 años, este rango aporta certeza y pertinencia al desarrollo de una aplicación móvil de RA, al ser una población que tiene la condición tanto de nativos cómo de migrantes digitales [Prensky 2001]. En cuanto a la información específica de la encuesta, se indagaron tres (3) aspectos fundamentales: los lugares que se debían considerar, los hechos 
X Congresso Brasileiro de Informática na Educação (CBIE 2021)

Anais do XXXII Simpósio Brasileiro de Informática na Educação (SBIE 2021)

históricos a incluir y los recursos multimedia disponibles; para la selección final de cada aspecto, se tomó aquellos que tuvieran el apoyo de cuatro (4) o más participantes de la encuesta.

En el primer aspecto, se presentó a los docentes algunos bienes inmuebles públicos junto con una opción abierta. Las respuestas obtenidas dejan entrever que todos los docentes entrevistados, consideran necesario incluir los lugares y monumentos más representativos históricamente para su enseñanza; para el caso de la ciudad de Pasto son: la calle del Colorado (100\%), el Parque Nariño (86\%), el Templo de San Juan, la Universidad de Nariño sede centro y El mono de la pila (todos con el 57\%). No se tuvo en cuenta en el aplicativo móvil de RA, lugares de carácter privado, puesto que se dificulta la consecución de información y permisos.

Con respecto a los hechos históricos que los docentes consideran más relevantes para su enseñanza, están sucesos como La Navidad Negra (100\%), la Transición de Plaza Mayor a Parque Nariño (86\%) y la Batalla de los Ejidos, Guerra de los Supremos y La Vida de Agustin Agualongo (todos con el 57\%).

En cuanto al tercer aspecto, todos los docentes encuestados creen muy importante incluir videos. Al 86\% le parece Muy Importante y un 14\% le parece Importante incluir representaciones 3D e imágenes. Con respecto a los audios y al texto, al $71 \%$ de la población le parece Muy Importante y al $29 \%$ les parece Importante agregarlos en el producto final. Se puede apreciar que estos cinco tipos de recursos educativos en una aplicación móvil de RA son valiosos, desde el punto de vista de los educadores en el campo de formación particular.

Por otra parte, para culminar con la apreciación de los docentes y dar mayor argumento a las decisiones tomadas, todos ellos confirman que si se llega a diseñar una aplicación móvil de RA con los requerimientos seleccionados, la usarían, expresando afirmaciones como: "Me ayudaría aproximarme a la realidad histórica de mi ciudad" o "Si, porque se convierte en una herramienta actual, moderna que rompe con las didácticas utilizadas hoy en día", entre otras, de modo que ésta pregunta del cuestionario indicó la intensión de uso por parte de los docentes.

\subsection{Diseño del Aplicativo Móvil de RA}

El diseño del aplicativo siguió las cinco (5) orientaciones propuestas por [Zuñiga 2008]: definición del aplicativo, definición de los sensores, definición del modelado semántico, definición de los dispositivos móviles y disponibilidad de información.

Definición del aplicativo: el tipo de aplicativo y el contexto de uso fueron definidos a partir de los requerimientos identificados con anterioridad. Se destaca que en la revisión de la documentación histórica, se encontraron los datos pertinentes dentro y fuera de la región, de los cuales se recopiló lo esencial, según los requerimientos que los encuestados (docentes) dieron a conocer, mencionados a continuación.

- El aplicativo debe permitir que el usuario acceda dinámica y atractivamente a la información de los lugares y hechos históricos de la ciudad.

- Desarrollar un aplicativo móvil con información de lugares y hechos históricos de la región, conforme la temática correspondiente en el currículo educativo. Para nuestro caso de uso, los docentes encuestados indicaron incluir los lugares y hechos históricos más representativos de la región, mencionados en la Subsección 4.1.

- El aplicativo móvil debe contar con variedad de recursos multimedia, principalmente videos, representaciones 3D e imágenes.

- Usar marcadores como tipo de RA, permitiendo así que el usuario no deba desplazarse al lugar histórico que desea visualizar y aprender.

- El aplicativo móvil debe contar con contenido offline, para que el usuario pueda acceder a la información, sin preocuparse por la conectividad. 


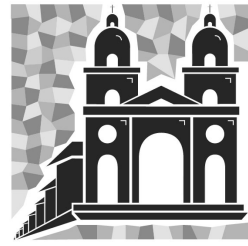

Figura 1. Marcador

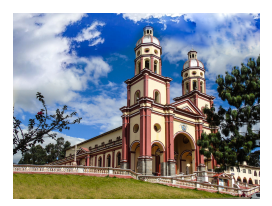

Figura 2. Ambiente real

- El aplicativo móvil debe contar con objetivos educativos que potencien el aprendizaje del estudiante y lo motiven a descubrir más información.

Definición de los dispositivos sensores: los dispositivos están sujetos al tipo de RA que se asuma; para el caso particular, el aplicativo móvil se ubica en el Nivel 1 de RA, donde se utilizan marcadores como herramienta de fácil acceso y bajo costo, que no requiere ubicar al usuario en el patrimonio histórico de la ciudad. En cuanto a hardware, requiere de un teléfono inteligente; con respecto al software, se debe pensar en móviles con un sistema operativo de uso genérico y bajo costo, en este caso se propone Android versión 5.0 o superior.

Definición del modelado semántico: se refiere al uso de vocabulario semántico para decodificación de los datos brutos obtenidos a través de los sensores; se utilizan etiquetas adecuadas y relevantes para la descripción de toda la información que se incluye en el aplicativo.

Definición de los dispositivos móviles: aquí se define el tipo de dispositivo móvil y la forma de procesamiento de información. Se usó el SDK de Vuforia, dado que ofrece un amplio nivel de compatibilidad con dispositivos móviles con versiones de Android 4 y superiores, permitiendo que la aplicación móvil este disponible a un mayor número de usuarios y por ende contribuir al aprendizaje del patrimonio histórico de la ciudad.

Disponibilidad de información: se refiere a que la información en la aplicación móvil sea contextual, esto se logra en la etapa de elicitación de requisitos. Con esta información clara y en cumplimiento de la sub etapa de "Definición de la aplicación", se dispone de información textual, fotografías, audios, videos y representaciones 3D de los lugares históricos de Pasto. También se resalta que, ante el requerimiento de disponibilidad de información offline, la aplicación posibilita el acceso a datos relevantes en cualquier momento.

\subsection{Implementación del Aplicativo Móvil de RA}

Con la información obtenida en las etapas anteriores, la implementación se realizó como sigue:

Creación e implementación de la estructura de la aplicación: se crea la estructura del proyecto en Unity junto con el SDK de Vuforia; se construyen tres escenas que separan los módulos del aplicativo: (1) una escena para navegar entre los diferentes lugares y monumentos de Pasto, que sería el "Menú principal"; (2) otra escena destinada a la información "Acerca de..." y (3) la escena principal para cada uno de los monumentos y lugares históricos escogidos. Esta escena incluye los diferentes recursos multimedia que sirven de apoyo para el aprendizaje histórico regional (fotografías, videos, audios, texto y el objeto 3D del lugar).

Creación de base de datos de imágenes objetivo: la base de datos fue creada en Vuforia, utilizando el tipo Device. Esta incluye los marcadores objetivo de la aplicación móvil, que consisten en representaciones minimalistas (Figura 1) del lugar al que cada una hace referencia (Figura 2), los lugares fueron vectorizados según las características principales del sitio.

Creación y recopilación del contenido tridimensional: el contenido 3D en su mayoría 
fue diseñado específicamente para el aplicativo, se utilizó el software 3D $\mathrm{Max}^{2}$ en su versión 2018 con licencia educativa, en cuanto a objetos de tipo mobiliario como árboles o imágenes religiosas, algunos fueron descargados de repositorios gratuitos ${ }^{3}$. Los audios, por su parte, tienen dos modalidades: los generados y los recopilados; los primeros son inéditos para el aplicativo, creados según la información textual recopilada, los segundos corresponden a podcast de diferentes autores. En cuanto a las imágenes, se hace la captura y recopilación de fotografías de los sitios escogidos de la ciudad para la ejecución del proyecto. Una vez realizados los pasos anteriores, el aplicativo de RA, denominado HistoryAR, es implementado y se procede a realizar la evaluación como se describe en la Sección 4.4.

\subsection{Evaluación y Resultados}

Se efectuó una evaluación por inspección del aplicativo, usando las Heurísticas de Nielsen [Nielsen 1995], con el fin de identificar posibles problemas de usabilidad, considerando que los usuarios finales serán estudiantes de octavo grado. A continuación, se describe cada una.

Visibilidad del estado del sistema: se identificó que el aplicativo no presentaba gran cantidad de información sobre el estado del sistema; por tanto, se procedió a realizar los ajustes necesarios para que el usuario pueda estar al tanto del funcionamiento del aplicativo. Se incluyeron elementos en la parte superior de la interfaz para mostrar, entre otros, el estado de la conectividad a Internet y la barra de precarga de videos (Figura 3), con símbolos fácilmente identificados por los estudiantes al interactuar con este tipo de elementos.

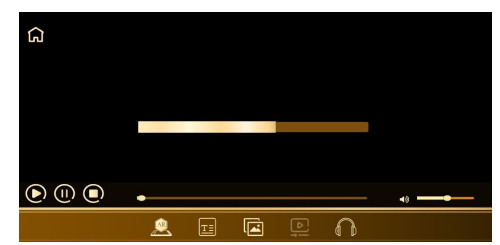

Figura 3. Ventana de carga del recurso multimedia (Video)

Coincidencia entre el sistema y el mundo real: esta heurística es cumplida al usar un lenguaje simple y una terminologia clara, tanto con el uso del idioma español como con términos contextuales y que son fácilmente reconocidos por los estudiantes que harán uso del aplicativo. Los videos y audios muestran la información acorde al nivel educativo de los estudiantes, en cuanto a terminología, conceptos e instrucciones.

Dar al usuario el control y la libertad: se evidenció que en el aplicativo el usuario tiene control y libertad en varios aspectos; por ejemplo, tiene la posibilidad de navegar entre los módulos de dos maneras diferentes, una es a través del menú lineal ubicado en la parte inferior de la pantalla principal y otra mediante el zoom (Figura 5) y exploración que se puede hacer en el mapa de la ciudad representada (Figura 4). Por otra parte, desde cualquier apartado del aplicativo, el usuario tiene a su disposición el botón de salida a la ventana principal; adicionalmente, al interior de cada módulo, están disponibles los recursos multimedia ya descritos, con los cuales se puede interactuar, esto indica que no es necesario un orden jerárquico para su uso, factor que es importante desde el punto de vista de aprendizaje y uso del aplicativo por parte de los estudiantes.

En cuanto a la información presentada con la tecnología de RA, el usuario puede acercar, alejar o mover el objeto 3D con movimientos típicamente usados al interactuar con otros

\footnotetext{
${ }^{2} \mathrm{https}$ ://www.autodesk.com/education/edu-software/overview?sorting=featuredpage $=1$

${ }^{3}$ https://free3d.com/; https://es.3dexport.com/
} 


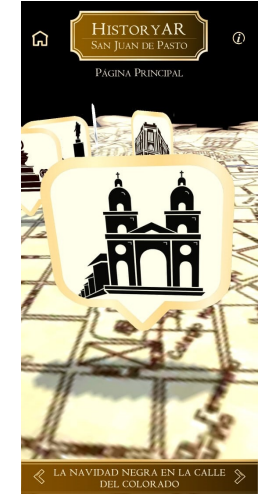

Figura 4. Acceso a módulos (menú)

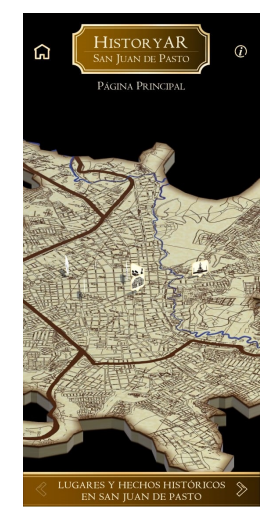

Figura 5. Acceso a módulos (zoom)

aplicativos en el celular. El usuario puede utilizar el movimiento de sus dedos sobre la pantalla del dispositivo o acercando y alejando el marcador según como desee.

Consistencia y estándares: el aplicativo móvil usa iconos que tienen una representación gráfica de su respectiva función, facilitando su uso y navegabilidad; por ejemplo, el icono de auriculares para el contenido multimedia de tipo audio o una casa para el botón que lleva a la página principal, en general, hay una puesta en juego pertinente de la iconografía.

Prevención de errores: se identificó que durante la navegación entre los módulos del aplicativo, uno de los errores que el usuario puede cometer, es equivocarse en la selección del lugar o situación histórica que desea consultar; por tanto, para remediar este tipo de errores, se utiliza la opción de retorno al menú principal, botón disponible en todos los módulos. En cuanto a los recursos multimedia de cada módulo, se cargan en la misma escena, así que cada botón de la parte inferior, permite activar o desactivar el recurso según se requiera.

Reconocer en lugar de recordar: considerando que el tamaño de una pantalla móvil es reducida comparada con la de un ordenador, un factor clave es la facilidad en la navegabilidad. Esta se hace por íconos con una representación gráfica del contenido al que hacen referencia, que los estudiantes pueden reconocer al instante mediante asociaciones de lo que es conocido por ellos; así, la interfaz gráfica del aplicativo, al tener un orden lógico y familiar, se convierte en una herramienta con funciones intuitivas para el usuario.

Flexibilidad y eficiencia de uso: se busca dar alternativas de usabilidad y navegabilidad en el aplicativo, utilizando la menor cantidad de recursos. Al respecto, en la página principal, se encuentran los lugares históricos seleccionados, estos se ubican sobre un mapa 3D de la ciudad, allí el usuario puede navegar entre los diferentes lugares mediante botones, esto también se puede hacer por la barra inferior que tiene los nombres de los lugares y monumentos históricos de la región. Adicionalmente, la orientación de la pantalla se adecúa de manera automática (Figura 3), según los recursos multimedia que se vayan a visualizar; por ejemplo, los videos suelen ser grabados en formato horizontal al igual que las fotografías, por tanto, es mejor que la orientación del dispositivo sea Landscape, mientras que la lectura de un texto es mejor en una orientación Portrait, de modo que así el aplicativo tiene una mejor eficiencia de uso.

Estética y diseño minimalista: toda la información y funciones incluidas en la aplicación móvil buscan cumplir una función clara y entendible; por una parte, están los botones, estos son vectores que representan su función o el contenido al que hacen referencia, de modo que el usuario fácilmente puede asociar el icono al recurso que desea visualizar; en cuanto a los marcadores que activan la RA en el aplicativo, son representaciones minimalistas del lugar histórico que representan y mantienen una estética acorde a la interfaz de todo el software. Por 
X Congresso Brasileiro de Informática na Educação (CBIE 2021)

Anais do XXXII Simpósio Brasileiro de Informática na Educação (SBIE 2021)

otra parte, la fuente seleccionada para los textos del aplicativo, los colores y tamaños fueron configurados de forma adecuados para la visualización del usuario.

Ayuda al usuario a reconocer, diagnosticar y recuperarse de los errores: el usuario en cualquier momento puede intercambiar entre los módulos o los recursos, si por algún momento se equivoca, tiene a su disposición el botón para ir al menú principal, lo cual le permitirá orientar nuevamente su recorrido a través del aplicativo. Adicionalmente, cuando existe una falla de conectividad a Internet, al usuario se le muestra un mensaje que indica qué recursos multimedia no estarán disponibles en ese estado; sin embargo, si el usuario restablece su conectividad y hace el llamado nuevamente al recurso, este se mostrará sin fallas y en su totalidad.

Ayuda y documentación: se concibe como principio básico en el desarrollo de todo software y en la RA esto no es la excepción. La ayuda disponible para orientar a los usuarios es un manual de usuario, que está disponible al momento que carga la página principal de HistoryAR, el cual puede ser omitido por los usuarios que así lo desean.

\section{Discusión}

La importancia del contexto, como requerimiento en la creación de aplicativos móviles de RA de carácter educativo - regional y los requerimientos para el desarrollo de este tipo de aplicativos con énfasis educativo - histórico, se convierten en líneas de acción para establecer que es fundamental la participación de expertos en educación del área; además, gracias a la evaluación heurística realizada, fue posible hacer los ajustes necesarios para cumplir con cada una de las reglas propuestas en ella.

En ese sentido, a partir de este estudio, se consolida un conjunto de requerimientos como una propuesta inicial para el diseño de un aplicativo móvil de RA que aporte en el aprendizaje del patrimonio histórico regional.

Enfoque educativo: es preciso tener información de primera mano acerca de los aspectos curriculares del área a abordar; las metas a alcanzar y los contenidos a trabajar se constituyen en aspectos relevantes que sólo los pueden dar los expertos del área, tras un buen proceso de elicitación. La didáctica, si bien es particular, se ve beneficiada con la puesta en marcha de recursos educativos digitales, acordes a la sociedad contemporánea.

Participación de actores involucrados: dar prioridad al conocimiento de los docentes del área para decidir el contenido del aplicativo a ser desarrollado, el cual estará acorde al nivel educativo y lineamientos curriculares. La participación de los usuarios finales [estudiantes] puede contribuir también al fortalecimiento del aplicativo de acuerdo a sus necesidades.

Determinar la finalidad: desde lo educativo e histórico se podría dar paso a que el aplicativo tenga un ciclo de vida más largo y tenga mayor soporte, en tanto que su uso será mayor y se debe mantener actualizado el conocimiento generado.

Identificar los ámbitos de aplicación: si bien todo se puede considerar histórico, hay algunos elementos más importantes que otros, que se deben identificar desde lo religioso, lo cultural y lo político. En este aspecto se deben tener en cuenta también los lineamientos curriculares acordes a los estudiantes que usarán el aplicativo.

Brindar accesibilidad: porque los aplicativos de RA puede ser "hermosos" pero los recursos pueden ser costosos, por tanto, mientras más sencillo y económico sea, más asequible es para cualquier persona.

Ofrecer información contextual: requerimiento que poco se tiene en cuenta en el diseño de aplicativos de RA, y van casi siempre a lo genérico. En este sentido, el acervo 
X Congresso Brasileiro de Informática na Educação (CBIE 2021)

Anais do XXXII Simpósio Brasileiro de Informática na Educação (SBIE 2021)

histórico regional debe ser contextual a nivel local, y esto se logra con la puesta en marcha de un buen proceso de elicitación de requisitos y con la participación de docentes correspondientes.

Priorizar la versatilidad y flexibilidad: este requerimiento le da especial importancia a tener contenido en condiciones de baja conectividad y considerar la poca movilidad del usuario; se debe sopesar lo que se almacena con lo que se muestra online, así como las condiciones que faciliten al usuario acceder a la información, aún sin estar presente físicamente en el sitio.

Variabilidad de recursos multimedia: incluir variabilidad de recursos con el fin de permitirle al estudiante conocer y profundizar el patrimonio histórico con las diferentes opciones multimedia.

Usabilidad: cumplir con los diferentes aspectos de usabilidad a fin de garantizar una adecuada interacción entre el usuario y la información digital, y así lograr que el usuario se apropie del contenido histórico presentado. Un aplicativo con alta usabilidad contribuirá en el cumplimiento de los objetivos para el cual este es desarrollado.

\section{Conclusiones}

Pese a que hoy en día existe un sin número de aplicativos móviles disponibles en Internet, aquellos destinados al aprendizaje histórico, y más aún que utilicen RA, son limitados; particularmente en la ciudad de Pasto, las investigaciones y software que tratan temas propios de la región, son destinados al turismo dejando de lado los fines educativos, aspecto que no se puede trabajar como se lo haría en otros ámbitos, puesto que demanda especial importancia de los contenidos, las metas educativas, las formas de comunicación y acceso a la información y, de la mano de esto, de la eficiencia, versatilidad y flexibilidad que le den al usuario la oportunidad de acceder sin mayores complicaciones.

En este sentido, el desarrollo de un aplicativo móvil de RA con fines educativos, requiere la contextualización del contenido a abordar con ayuda de la población que lo utilizará; esto se constituye en requerimiento fundamental que le da contexto al producto. En este ámbito, toma importancia la participación de docentes, tanto en contenidos académicos como en metas educativas a alcanzar, se configura en la base del aplicativo que le dará precisión a lugares, hechos y recursos a utilizar e implementar.

Otro aspecto que toma relevancia, es la puesta en marcha de la evaluación heurística, puesto que permite identificar posibles problemas de usabilidad que le darían baja calidad al producto; esto da paso a la revisión de las condiciones más particulares previas a su lanzamiento.

Los avances que se han obtenido en el desarrollo del presente producto, permiten proponer una serie de requerimientos, que están puestos para discusión y que se postulan como consideraciones relevantes al momento de desarrollar un aplicativo móvil de RA, que aporte en el aprendizaje del patrimonio histórico regional.

Finalmente, es pertinente expresar que, a futuro, se pretende hacer una evaluación empírica con estudiantes de grado octavo de la ciudad de Pasto, mediante la técnica "Test Piense en Voz Alta", lo que permitirá efectuar una observación directa sobre la muestra de usuarios [estudiantes] seleccionados de forma aleatoria, donde se evidencie su interacción con el aplicativo móvil de RA; posteriormente, los usuarios responderán un cuestionario con preguntas abiertas, donde expondrán su nivel de satisfacción frente al uso del aplicativo; este proceso se llevará a cabo a fin de hacer ajustes necesarios a los requerimientos propuestos, así como identificar nuevos aspectos a ser considerados para el tipo de aplicativo aquí indicado. 
X Congresso Brasileiro de Informática na Educação (CBIE 2021)

Anais do XXXII Simpósio Brasileiro de Informática na Educação (SBIE 2021)

\section{Referências}

Aliprantis, J. and Caridakis, G. (2019). A survey of augmented reality applications in cultural heritage. International Journal of Computational Methods in Heritage Science (IJCMHS), 3(2):118-147.

Bekele, M. K., Pierdicca, R., Frontoni, E., Malinverni, E. S., and Gain, J. (2018). A survey of augmented, virtual, and mixed reality for cultural heritage. Journal on Computing and Cultural Heritage (JOCCH), 11(2):1-36.

Bonifaz, E. and Molina, F. (2015). Realidad aumentada y su aporte al patrimonio cultural. In I Congreso Internacional de Salvaguardia y preservación del patrimonio cultural.

Cardenas, M. I. Z. (2020). Vítica, patrimonio y cultura: Producción de contenidos digitales para realidad aumentada. Sphera Publica, 1(20):9-33.

Dresch, A., Lacerda, D. P., and Júnior, J. A. V. A. (2015). Design science research: método de pesquisa para avanço da ciência e tecnologia. Bookman Editora.

Fraga, T. and de Menezes, C. (2017). Um ambiente para autoria e realização de aplicações educacionais com realidade aumentada. In Brazilian Symposium on Computers in Education (Simpósio Brasileiro de Informática na Educação-SBIE), volume 28, page 937.

Hincapié, M., Díaz, C., and Valencia, A. (2020). Usando realidad aumentada para el aprendizaje del patrimonio histórico y cultural, chapter 3, pages 44-52. Sello Editorial Coruniamericana.

Luna, U., Ibáñez, A., and Rivero, M. P. (2019). El patrimonio aumentado. 8 apps de realidad aumentada para la enseñanza-aprendizaje del patrimonio. Revista interuniversitaria de formación del profesorado, (94):43-62.

Martinez Carrillo, A. L. et al. (2021). La realidad aumentada como recurso didáctico para la enseñanza y el aprendizaje del patrimonio histórico. el edifico del mosaico de los amores del conjunto arqueológico de cástulo (linares, jaén). UNES Universidad Escuela y sociedad, (10):32-43.

Mejía, J., Muñoz, Z., and Quiróz, C. (2012). Juega, diviértete y aprende con los personajes y escenarios más representativos del departamento de "nariño" que intervinieron en la época de independencia (1809-1825), recurso educativo 3d. Universidad de Nariño.

Meneses, J. and Montenegro, F. (2015). Sistema de realidad aumentada para promover y enriquecer atractivos turísticos del centro histórico de la ciudad de pasto "turistar". Universidad de Nariño.

Moran, C. and Narvaez, A. (2015). Pasto ar aplicación móvil para la promoción turística de la ciudad de pasto con realidad aumentada para android. Universidad de Nariño-SIRED.

Nagata, J. J., Abad, F. M., and Giner, J. R. G.-B. (2017). Realidad aumentada y navegación peatonal móvil con contenidos patrimoniales: Percepción del aprendizaje. RIED. Revista Iberoamericana de Educación a Distancia, 20(2):93-118.

Nielsen, J. (1995). 10 usability heuristics for user interface design. Nielsen Norman Group, 1.

Pimentel, M., Filippo, D., and Santoro, F. M. (2019). Design science research: fazendo pesquisas científicas rigorosas atreladas ao desenvolvimento de artefatos computacionais projetados para a educação. Metodologia de Pesquisa em Informática na Educação: Concepção da Pesquisa. Porto Alegre: SBC.

Prensky, M. (2001). Digital natives, digital immigrants. On the Horizon, 9(6):1-6. 
Anais do XXXII Simpósio Brasileiro de Informática na Educação (SBIE 2021)

Rodríguez, E., Otero, J., Puello, J., and Orozco, Z. (2016). Capítulo 6. una alternativa tecnológica para el aprendizaje y apropiación patrimonial del museo histórico de cartagena. In Recursos Educativos Aumentados Una Oportunidad para la Inclusión, pages 66-73.

Suárez, J. (2017). Geohistoryapp: Realidad aumentada basada en geolocalización para aprender historia. Universidad Distrital Francisco José de Caldas.

Torres, D. R. (2011). Realidad aumentada y patrimonio cultural: nuevas perspectivas para el conocimiento y la difusión del objeto cultural. E-rph: Revista electrónica de Patrimonio Histórico, (8):92-113.

Zuñiga, J. C. (2008). Uma metodologia para o desenvolvimento de aplicações de realidade aumentada em telefones celulares utilizando dispositivos sensores. PhD thesis, Universidade de São Paulo. 